\title{
Distributed Association Control in Shared Wireless Networks
}

\author{
Krishna C. Garikipati, Kang G. Shin \\ Department of Electrical Engineering and Computer Science \\ University of Michigan-Ann Arbor \\ Email: \{gkchai, kgshin\}@eecs.umich.edu
}

\begin{abstract}
In modern wireless networks, fairness of user throughputs is often important to efficiently utilize the available network bandwidth. Association control-control of user associations to reduce overloading of access points (APs) - has been the standard approach to address this problem. However, in the recent paradigm of shared wireless networks in both, community and commercial space, access points are increasingly managed without any central coordination. As a result, achieving user level fairness in these settings remains a challenging task.

In this paper, we consider the global utility of proportional fairness. Specifically, we propose a distributed approach to association control, in which each user makes association decisions based on the information gathered from probe responses from its nearby APs. Two solutions are presented: (i) a randomized approach, in which users update their associations probabilistically, and (ii) a deterministic association rule that converges quickly. We provide theoretical guarantees on the performance of both approaches. Our simulation results show that the proposed solutions can significantly improve fairness and overall throughput compared to other association heuristics.
\end{abstract}

\section{INTRODUCTION}

The prolific growth in wireless technologies over the last decade can be gauged by today's dense deployment of WiFi networks in homes, offices and community hotspots. At the same time, the low cost and popularity of wireless devices has led to the paradigm of Shared Wireless Networks (also known as Wireless Community Networks). In these networks, access points function in the 'open' mode and provide connectivity to users who do not necessarily have ownership over them. Such open-source model of wireless access has many advantages, like using under-utilized access points for possible monetary gains, increased network capacity and improved coverage for mobile users. Two sharing models have emerged. Peer-to-peer sharing, facilitated by commercial ventures, e.g. FON [1], where private access points are available to registered public users for free or in a quid pro quo manner so that users who register also open up their access point in return. And, Public sharing, in hotspot deployments of cellular service providers and through community initiatives [2], [3]. Given the tremendous benefits, sharing of WiFi networks in urban areas is proposed as an inexpensive alternative to cellular networks.

The underlying economics of sharing access points is an interesting problem. Previous research has studied the various models for incentive mechanisms and access schemes for wireless community networks [4]. In our work, we assume a shared wireless network is in place and instead focus on the important problem of user association with access points (APs). Although fairness-aware user associations has been studied in similar deployments of WLANs, they are fundamentally tied to the presence of a central controller. In this work, we present a distributed solution for user associations that assumes no global coordination.

The default AP-association of users can lead to some highly congested APs while leaving the rest of the APs under-loaded with few users connected to them. The overloading of APs not only causes unbalanced users' throughput (hence unfairness) but also lowers the overall system throughput. This is observed in most deployments of Mesh, WLANs and open WiFi networks [5], [6], [7]. In practice, however, the association metric implemented in wireless devices only considers the RSSI (received signal strength indicator) values for AP selection. Consequently, a number of heuristic based association schemes [5], [8], [9] that are distributed and maximize individual user throughputs have been proposed in literature. On the other hand, centralized approach to user associations in WLANs addresses the problem of fair throughput allocation [6], [10], [11]. In this work, we show that the global objective of fairness can also be achieved in a completely distributed way, a key requirement for shared wireless networks. In the following, we discuss some key features of our model.

Association control: The concept of association control [6] was introduced to achieve load-balancing of APs. Following this mechanism, users are switched and reassociated with different APs so as to maximize a fairness measure of the users' throughput. By using the notion of max-min fairness, the authors showed that the solution of the problem balances the APs' loads for an appropriately defined load metric. However, as pointed out later, the objective of max-min fairness can lead to low aggregate throughputs [12]. To balance between fairness and throughput, the proportional fair utility was used to obtain optimal user associations [10], [11]. The solution required solving a discrete optimization problem using integer relaxation. All of these approaches assume a central server that solves the optimization problem offline and a periodic update mechanism to switch users between APs [6], [11]. Clearly, such an assumption fails to hold in our setting where sharing emerges on an ad-hoc basis.

Uncoordinated APs: Excluding enterprise WLAN deployments, access points are mostly installed as plug-and-play devices, and the configurations are done at a local level. For 


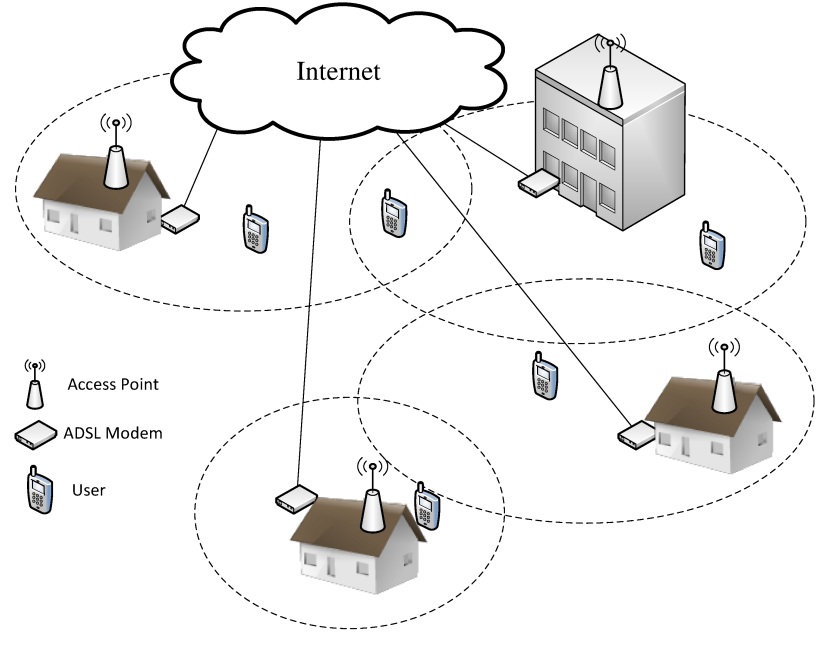

Fig. 1. Example of a shared WiFi network [1] where APs are uncoordinated and have a limited backhaul capacity

example, in a shared wireless network like FON, access points are set up by individual owners. The assumption of uncoordinated APs is also reasonable in large scale WLANs, for e.g. public hotspots, where cost and scalability of WLAN controller remains an issue. In the absence of global information, the APs need to select their transmission channel, transmission power and user-associations. This will generally result in low user throughputs due to interference and uneven user loads on the APs. Previous work [13], [14] described these settings under Self-Organizing WiFi networks where the channels of APs and user associations are managed autonomously. A distributed method based on Gibbs sampling [15] was proposed for the user-association problem. Nevertheless, the objective function used gave no insights in system performance.

Backhaul limited: An important architectural feature of community and open WiFi hotspot deployments is the limited backhaul capacity of the access points [7], [16]. In fact, the current wireless standards $802.11 \mathrm{~g}$ and $802.11 \mathrm{n}$ offer nominal throughputs up to $54 \mathrm{Mbps}$ and $300 \mathrm{Mbps}$, respectively. Even with the contention and transmission overhead, the throughput is an order-of-magnitude greater than the bandwidth of ADSL/cable connections. As a result, the maximum throughput available from APs deployed in homes/offices is limited by the capacity of the backhaul link. Also, in deployments where APs are connected via a wireless mesh network, e.g., Municipal WiFi networks, it is possible the wireless link (or route) between the AP and the mesh gateway can become a bottleneck. Therefore, user associations in general network deployments should consider the backhaul capacity.

The above aspects are illustrated in Fig. 1. The users share a common set of APs deployed in a residential area over a backhaul of ADSL cable connections. Since there is no central coordination in the network, AP-selection decisions need to be made by users themselves. We consider the association control problem that arises in this scenario. In particular, we are interested in the global objective of proportional fairness (PF) of user throughputs. As the optimization problem is NP-hard, we present a randomized solution where users associate with each AP following a probability rule. We show that the randomized solution can be implemented in a distributed fashion and also include the practical overhead of scanning and beacon losses. We then apply a fast converging heuristic to the randomized solution. As the associations need not be optimal, bounds are provided in special cases. To the best of our knowledge, our approach is the first to present a distributed association control in wireless access networks.

The remainder of the paper is organized as follows. Section II lays out the notation used and formulates the optimization problem. In Section III, we give a randomized solution to this problem. In Section IV, a deterministic analogue of the randomized association scheme is presented. Section V evaluates the performance of these solutions and compares it with other existing association heuristics. Finally, the paper concludes with Section VI.

\section{SYSTEM MODEL}

\section{A. Notation}

Consider a wireless network with a set of APs, $\mathcal{S}:=$ $\{1,2 \ldots, S\}$ and a set of mobile users, $\mathcal{N}:=\{1,2 \ldots, N\}$. Assume that user $i, i \in \mathcal{N}$ can associate with a single AP from a subset of APs, $\mathcal{S}_{i} \subseteq \mathcal{S}$, depending on its location, received signal strength and preferences. We implicitly let $N>1$ and $\left|\mathcal{S}_{i}\right| \geq 1$ for all users $i \in \mathcal{N}$. The set of all possible associations, $\mathcal{F}=\prod_{i} S_{i}$ is called the feasible set. Let us denote the association of user $i \in \mathcal{N}$ by $a_{i} \in S_{i}$ and define the association vector $\mathbf{a}:=\left(a_{i}\right)_{i \in \mathcal{N}}$, indexed by the users. Also, let $n_{s}(\mathbf{a}):=\left\{i \in \mathcal{N} \mid a_{i}=s\right\}$ be the set of users connected to an AP $s \in \mathcal{S}$. We use the notation $\mathbf{a}_{-i}=\left(a_{1}, \ldots a_{i-1}, a_{i+1}, \ldots a_{N}\right)$ to represent the association vector of all users except $i$ and $\left(b_{i}, \mathbf{a}_{-i}\right)$ to denote the vector $\left(a_{1}, \ldots a_{i-1}, b_{i}, a_{i+1}, \ldots a_{N}\right)$. Unless specified otherwise, we make the following assumptions about our model.

- The users $\mathcal{N}$, and their feasible associations, $\mathcal{S}_{i}$, for all $i \in$ $\mathcal{N}$ are fixed, i.e., there is no new user arrival or departure and the location of users does not change.

- Users are cooperative, i.e., they have a common objective function and behave in a non-strategic way to achieve an optimal state.

- Association control is done over a longer time-scale and ignores short-term effects like channel fading

\section{B. Throughput model}

For a given association, $\mathbf{a} \in \mathcal{F}$, of users in the network, let $r_{i}(\mathbf{a})$ be the throughput user $i \in \mathcal{N}$ receives from its chosen AP, $a_{i} \in \mathcal{S}_{i}$. As data flows and channel characteristics are varying in nature, we restrict our attention to the long-term throughputs. Moreover, association policies based on instantaneous throughput will not be stable. Let $B_{i, a_{i}}>0$ be the PHY-transmission rate of user $i \in \mathcal{N}$. This is the rate at which the AP or user transmits data when the user is scheduled. It takes only discrete 
values given by the rate-adaptation algorithm of $802.11 \mathrm{MAC}$ and is a function of the Signal-to-Noise ratio (SNR) of the link.

When two or more APs share a common channel and lie in the same collision domain, each AP will gain access to the channel for a fraction of time. For AP $s \in \mathcal{S}$, this fraction is denoted by $f_{s} \in(0,1)$. Consider the case where all user dataflows are homogeneous and fully saturated, and that an AP always has a packet to trasnmit. Assuming round-robin scheduling with infinite buffer size, the downlink throughput of user $i \in \mathcal{N}$ is given by

$$
r_{i}(\mathbf{a})=\frac{f_{a_{i}}}{\sum_{j \in n_{a_{i}}(\mathbf{a})} 1 / B_{j, a_{i}}+O\left(\left|n_{a_{i}}(\mathbf{a})\right|\right)}
$$

where the overhead term $O\left(\left|n_{a_{i}}(\mathbf{a})\right|\right)$ accounts for MAC layer overhead like ACKs. The overhead term only depends on number of users, $\left|n_{a_{i}}(\mathbf{a})\right|$, assuming equal overhead for all users. Note that the long-term throughput of all users $i \in n_{a_{i}}(\mathbf{a})$ connected to AP $a_{i}$ is equal due to fair scheduling. A similar expression is known for the uplink throughput and is attributed to the rate anomaly of 802.11 DCF [17].

Consider the case where each AP $s$ has a finite backhaul link capacity $W_{s}>0$ determined by the backbone infrastructure, e.g., ISP. This gives an upper limit on the maximum bandwidth available to the users connected to the AP. Assume this bandwidth is equally shared among the users. Accordingly, the actual throughput of a user is the minimum of the capacity available from the wired and the wireless link [6].

$r_{i}(\mathbf{a})=\min \left\{\frac{f_{a_{i}}}{\sum_{j \in n_{a_{i}}(\mathbf{a})} 1 / B_{j, a_{i}}+O\left(\left|n_{a_{i}}(\mathbf{a})\right|\right)}, \frac{W_{a_{i}}}{\left|n_{a_{i}}(\mathbf{a})\right|}\right\}$

The above expression also holds for uplink traffic of users. Therefore, throughput definition of a user could refer to either uplink or downlink.

\section{Objective function}

The association control problem is to find user associations that maximize a global objective function. Here, we define the global utility, $U: \mathcal{F} \rightarrow \mathbb{R}$, to be the sum of logarithm of user throughputs as follows.

$$
U(\mathbf{a}):=\sum_{i \in \mathcal{N}} \log r_{i}(\mathbf{a})
$$

The utility is well defined since $r_{i}(\mathbf{a})>0$ for all users $i \in \mathcal{N}$ and all $\mathbf{a} \in \mathcal{F}$. Also, from the throughput expression of a user in Eq. (2), we can further express Eq. (3) as $U(\mathbf{a})=\sum_{s \in \mathcal{S}} U_{s}\left(n_{s}(\mathbf{a})\right)$, where the utility of each AP $s \in S$ is defined by $U_{s}\left(n_{s}(\mathbf{a})\right):=\sum_{j \in n_{s}(\mathbf{a})} \log r_{j}(\mathbf{a})$ and $U_{s}(\emptyset):=0$. We call the user associations that maximize the global utility as proportionally fair. Therefore, the problem is to solve the following combinatorial optimization problem:

$$
\max \{U(\mathbf{a}), \mathbf{a} \in \mathcal{F}\}
$$

Let $\mathcal{F}^{\star} \subseteq \mathcal{F}$ be the set of optimal solutions and OPT be the optimal value. In the following sections, we detail approaches to efficiently solve this problem.

\section{RANDOMIZED SOLUTION}

The optimization problem in Eq. (4) is intractable due to the combinatorial nature, but can be solved through a general approach of randomization. The basic idea is to associate users with APs probabilistically, where each user $i \in \mathcal{N}$ connects to an AP in the set $\mathcal{S}_{i}$ for a random duration, probes nearby APs in its range and switches to another randomly chosen AP. As this results in a random process of user associations, by choosing an appropriate steady state distribution, we ensure that the optimal solutions are the most likely to occur.

To this end, let the time begin at $t=0$ and assume the initial association of users is given. Our objective is to generate a continuous-time Markov chain, $\{X(t)\}_{t \in \mathbb{R}_{+}}$, of user associations that has a stationary distribution $\pi, \pi: \mathcal{F} \rightarrow[0,1]$, given by

$$
\pi(\mathbf{a})=\frac{\exp (\beta U(\mathbf{a}))}{\sum_{\mathbf{a}^{\prime} \in \mathcal{F}} \exp \left(\beta U\left(\mathbf{a}^{\prime}\right)\right)}, \quad \mathbf{a} \in \mathcal{F}
$$

where $\beta>0$ is a fixed parameter. This distribution, also known as Gibbs measure [15], favors association vectors with large objective values. By tuning the parameter, $\beta$, the distribution converges to the optimal solutions as shown below.

Lemma 1. For every $\mathbf{a} \in \mathcal{F}^{\star}, \pi(\mathbf{a})$ is an increasing function in $\beta$. Moreover, as $\beta \rightarrow \infty, \pi(\mathbf{a})=\frac{1}{\left|\mathcal{F}^{\star}\right|}, \forall \mathbf{a} \in \mathcal{F}^{\star}$.

Proof: See Appendix.

The transitions in the process, $\{X(t)\}_{t \in \mathbb{R}_{+}}$, correspond to change in state of user associations. To facilitate analysis, as will be clear later, we require that only a single user in the network changes its association at a time. This requirement can be practically realized by slightly modifying the reassociation procedure of mobile users.

\section{A. Update process}

The IEEE 802.11 standard [18] defines handoff as a process through which a user changes association between APs. It involves active/passive scanning of APs on different channels, sending association requests, authentication and higher layer processes like DHCP requests, IP route changes, etc. Although, a handoff is generally triggered when a user moves from the coverage of one AP to another, it can also be initiated to select APs in a proactive manner. This feature is implemented in most wireless drivers (e.g., MadWifi [19]) through periodic background scans. In our case, we relax the periodicity constraint and assume the scans are triggered according to a Poisson process with rate $\lambda>0$. That is, each user implements a clock with exponential i.i.d. inter-tick intervals and at each clock tick, it possibly reassociates with another AP. As the probability of two or more clock ticks occurring at the same instant is zero, association of only a single user is updated at a time. The associations in system then describe a continuous time Markov chain $\{X(t)\}_{t \in \mathbb{R}_{+}}$. Here, we have assumed the overhead in scanning and changing association is $<<1 / \lambda$, so that the tick instant is also the instant of reassociation. This is 
reasonable because a scan is triggered in the order of minutes while reassociation of users takes around the order of $100 \mathrm{~ms}$.

The continuous time process, $\{X(t)\}_{t \in \mathbb{R}_{+}}$, can be further discretized as follows: The overall ticks in the system is a Poisson process, $\{Z(t)\}_{t \in \mathbb{R}_{+}}$, with rate $N \lambda$. Denote the global ticks by $T_{k}, k=1,2 \ldots$ and $T_{0}=0$, and map the interval $\left[T_{k}, T_{k+1}\right)$ to the $k$-th time-slot. The equivalent discrete-time Markov chain, $\left\{A_{k}\right\}_{k \in \mathbb{Z}_{+}}$with state space $\mathcal{F}$ has the same stationary distribution given by Eq. (5), where the equivalence follows from the relation, $X(t)=A_{Z(t)}, t \in \mathbb{R}_{+}$.

\section{B. Gibbs sampler}

Gibbs sampling [15] is a classical MCMC method to simulate a markov chain with known stationary distribution. In our setting, the markov chain is $\left\{A_{k}\right\}_{k \in \mathbb{Z}_{+}}$, and the steady state is described by (5). Using this method, we show our main result that user associations can be updated in a completely distributed and asynchronous fashion.

Since all users implement an exponential clock with the same rate, it is equally likely that a global clock tick belongs to a particular user. Let the current association state be $\mathbf{a} \in \mathcal{F}$ and let the global clock be triggered by user $i \in \mathcal{N}$. The user $i$ updates its association to another randomly chosen $\mathrm{AP} b_{i}$ from the set $\mathcal{S}_{i}$ as follows:

$$
p\left(b_{i} \mid \mathbf{a}\right)=\frac{\exp \left(\beta U\left(b_{i}, \mathbf{a}_{-i}\right)\right)}{\sum_{s \in \mathcal{S}_{i}} \exp \left(\beta U\left(s, \mathbf{a}_{-i}\right)\right)}
$$

Note that it is possible to select $b_{i}=a_{i}$, in which case the user $i$ simply continues with its current association. The corresponding one-step transition probability of the Markov chain $\left\{A_{k}\right\}_{k \in \mathbb{Z}_{+}}$between states $\left(a_{i}, \mathbf{a}_{-i}\right)$ and $\left(b_{i}, \mathbf{a}_{-i}\right)$ is $P\left(\left(a_{i}, \mathbf{a}_{-i}\right) \rightarrow\left(b_{i}, \mathbf{a}_{-i}\right)\right)=\frac{1}{N} p\left(b_{i} \mid \mathbf{a}\right)$ for $b_{i} \neq a_{i}$. We note that $P\left(\mathbf{a} \rightarrow \mathbf{a}^{\prime}\right)=0$ whenever states $\mathbf{a}, \mathbf{a}^{\prime} \in \mathcal{F}$ differ in more than one coordinate.As the probability of visiting any state in $\mathcal{F}$ is positive, and self-looping is allowed, the Markov chain $\left\{A_{k}\right\}_{k \in \mathbb{Z}_{+}}$is both irreducible and aperiodic. Furthermore, it is easy to verify that $\pi(\cdot)$ is the unique stationary distribution.

Although, the above procedure gives us the desired steady state distribution, it is still centralized in its current form. In particular, to compute the transition probability in Eq. (6), user $i \in \mathcal{N}$ needs to know the value of the global utility $U\left(b_{i}, \mathbf{a}_{-i}\right)$, for all possible $b_{i} \in \mathcal{S}_{i}$. Let us define

$$
\phi_{i}\left(b_{i}, \mathbf{a}_{-i}\right):=U_{b_{i}}\left(n_{b_{i}}\left(b_{i}, \mathbf{a}_{-i}\right)\right)-U_{b_{i}}\left(n_{b_{i}}\left(\mathbf{a}_{-i}\right)\right)
$$

where we have used the notation $n_{b_{i}}\left(\mathbf{a}_{-i}\right):=\{j \in \mathcal{N}, j \neq$ $\left.i \mid a_{j}=b_{i}\right\}$ to denote the set of users not including $i$ associated with AP $b_{i}$. Then, using the separability of the objective function, we can further simplify Eq. (6) as

$$
\begin{aligned}
p\left(b_{i} \mid \mathbf{a}\right) & \propto \exp \left(\beta U\left(b_{i}, \mathbf{a}_{-i}\right)\right) \\
& \propto \exp \left[\beta\left(U_{b_{i}}\left(n_{b_{i}}\left(b_{i}, \mathbf{a}_{-i}\right)\right)+\sum_{s \in \mathcal{S}-\{b\}} U_{s}\left(n_{s}\left(\mathbf{a}_{-i}\right)\right)\right)\right] \\
& \propto \exp \left[\beta\left(U_{b_{i}}\left(n_{b_{i}}\left(b_{i}, \mathbf{a}_{-i}\right)\right)-U_{b_{i}}\left(n_{b_{i}}\left(\mathbf{a}_{-i}\right)\right)\right)\right] \\
& \propto \exp \left[\beta \phi_{i}\left(b_{i}, \mathbf{a}_{-i}\right)\right]
\end{aligned}
$$

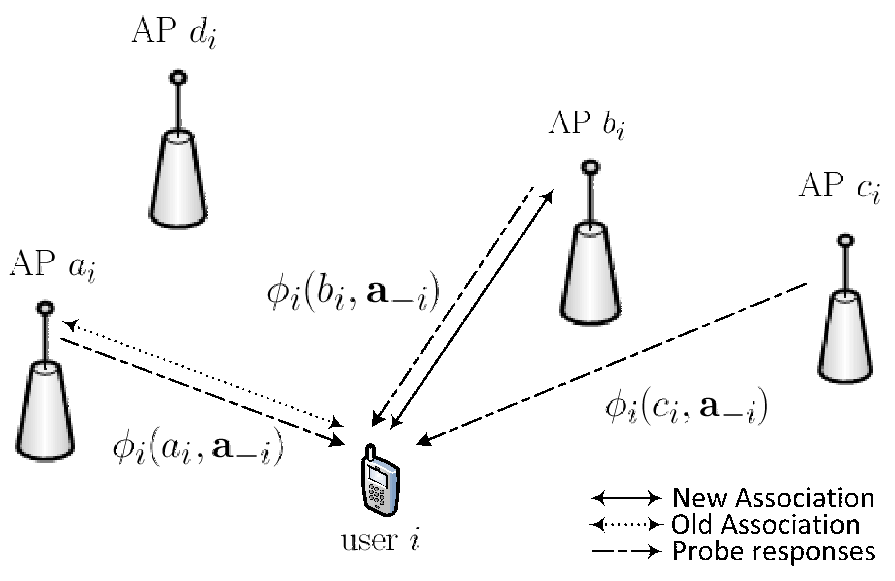

Fig. 2. An illustration of the process of changing associations based on probe responses obtained from nearby APs

and therefore,

$$
p\left(b_{i} \mid \mathbf{a}\right)=\frac{\exp \left[\beta \phi_{i}\left(b_{i}, \mathbf{a}_{-i}\right)\right]}{\sum_{s \in \mathcal{S}_{i}} \exp \left[\beta \phi_{i}\left(s, \mathbf{a}_{-i}\right)\right]} .
$$

The function, $\phi_{i}\left(b_{i}, \mathbf{a}_{-i}\right)$ is the marginal utility of user $i \in$ $\mathcal{N}$ w.r.t. the access point $b_{i} \in \mathcal{S}_{i}$. It is equal to the change in the utility of an AP due to the addition of the user. Now, each AP $b_{i}$ will know the PHY rates $B_{j, b_{i}}$ of its currently associated users $j \in n_{b_{i}}\left(\mathbf{a}_{-i}\right)$. Also, the fractional airtime $f_{b_{i}}$ and backhaul bandwidth $W_{b_{i}}$ can be known from simple passive measurements. The overhead term, on the other hand, can be computed from a known expression [17]. Consequently, the value of $\phi_{i}\left(b_{i}, \mathbf{a}_{-i}\right)$ can be calculated by AP $b_{i}$ using Eqs. (2) and (3), and each user only needs to obtain the marginal utilities from the neighborhood APs. Therefore, the update equation in Eq. (8) allows a completely distributed mechanism for user association where each user needs to obtain information locally.

The process of obtaining marginal utilities from APs is asymmetric in practice. Since a user $i \in \mathcal{N}$ is already associated with AP $a_{i}$, it will know $\phi_{i}\left(a_{i}, \mathbf{a}_{-i}\right)$ through direct communication with the AP. However, to obtain the rest of the marginal utilities, a user has to setup explicit communication channels with each AP in $\mathcal{S}_{i} \backslash\left\{a_{i}\right\}$. This is achieved through the process of active scanning, as specified in the 802.11 standard [18]. Assume the information about the channel ID and the MAC address of the surrounding APs is available to a user. The scanning is performed as follows. A user suspends its transmission state and switches to the channel of AP it wants to probe. It then sends a unicast probe request frame and waits for a probe response before switching to the next channel. This is repeated until all channels have been scanned. The collected probe responses contains information about user's marginal utility, based on which, the user chooses its next association (Fig. 2).

\section{Partial information}

The randomized association of users has an inherent overhead in terms of the number of message exchanges. In particular, a user needs to probe all of $\left|\mathcal{S}_{i}\right|-1$ surrounding APs at every clock trigger. This causes a scanning delay of the 
order of hundreds of milliseconds and affects real-time traffic. In addition, there is a significant reassociation (if triggered) overhead in the order of seconds that will disrupt user traffic. To avoid the excessive scanning delay, we consider the case where only a subset of APs are probed by a user during the scan phase. Also, it is possible that some of the probe responses from the APs get delayed, discarded or dropped [14]. Collectively, this implies that a user has access to a partial subset of all marginal utilities.

Let $T_{i} \subseteq \mathcal{S}_{i}$ denote the set of APs from which the marginal utilities are available to user $i$ after the probing process. The set $T_{i}$ is always non-empty because the marginal utility of the current $\mathrm{AP}, \phi_{i}\left(a_{i}, \mathbf{a}_{-i}\right)$, is assumed to be known. User association is now updated based on the available information, in a manner similar to Eq. (8):

$$
p\left(b_{i} \mid \mathbf{a} ; T_{i}\right)= \begin{cases}\frac{\exp \left(\beta \phi_{i}\left(b_{i}, \mathbf{a}_{-i}\right)\right)}{\sum_{s \in T_{i}} \exp \left(\beta \phi_{i}\left(s, \mathbf{a}_{-i}\right)\right)}, & \text { if } b_{i} \in T_{i} \\ 0 & \text { o.w. }\end{cases}
$$

Here, we assume a user will not be associated with an AP if the marginal utility from it is not available. Assume the event that a marginal utility from an AP $b_{i} \neq a_{i}$ is known to a user $i$ occurs independently in each scan of the user. The independence could represent situations of random loss of probe frames or when a user decides to randomly pick a subset of APs to probe after each clock trigger. Let $q_{i, b_{i}} \in(0,1]$ denote the probability of this event and let $\left\{A_{k}^{\prime}\right\}_{k \in \mathbb{Z}_{+}}$be the Markov chain generated from the resulting associations.

Theorem 1. The Markov chain $\left\{A_{k}^{\prime}\right\}_{k \in \mathbb{Z}_{+}}$is irreducible, aperiodic and the unique stationary distribution is given by:

$$
\pi^{\prime}(\mathbf{a})=\frac{\eta_{\mathbf{a}} \exp (\beta U(\mathbf{a}))}{\sum_{\mathbf{a}^{\prime} \in \mathcal{F}} \eta_{\mathbf{a}^{\prime}} \exp \left(\beta U\left(\mathbf{a}^{\prime}\right)\right)}, \quad \mathbf{a} \in \mathcal{F}
$$

where $\eta_{\mathbf{a}}=\prod_{i \in \mathcal{N}} q_{i, a_{i}}$

Proof: We only verify that the detailed balanced equations hold as the rest of the properties are straightforward to show. Consider any two distinct states, $\left(a_{i}, \mathbf{a}_{-i}\right)$ and $\left(b_{i}, \mathbf{a}_{-i}\right)$ for any $i \in \mathcal{N}$ and $b_{i}, a_{i} \in \mathcal{S}_{i}$. Then, $P\left(\left(a_{i}, \mathbf{a}_{-i}\right) \rightarrow\left(b_{i}, \mathbf{a}_{-i}\right)\right)=\frac{1}{N} \sum_{T_{i}: T_{i} \subset \mathcal{S}_{i}} P\left(T_{i}\right) p\left(b_{i} \mid \mathbf{a} ; T_{i}\right)$, where the summation is over all possible subsets of $\mathcal{S}_{i}$, and $P\left(T_{i}\right)$ is the probability that marginal utilities from APs in $T_{i}$ are known after a scanning process. We have, $P\left(T_{i}\right)=\prod_{s \in T_{i}} q_{i, s} \prod_{s \in \mathcal{S}_{i} \backslash T_{i}}\left(1-q_{i, s}\right)$. Therefore, for $b_{i} \in$ $T_{i}, P\left(T_{i}\right) \propto q_{i, b_{i}}$. Also, from the user update rule in Eq. (8), $p\left(b_{i} \mid T_{i}\right) \propto \exp \left(\beta \phi_{i}\left(b_{i}, \mathbf{a}_{-i}\right)\right)$. Therefore, we can write $P\left(\left(a_{i}, \mathbf{a}_{-i}\right) \rightarrow\left(b_{i}, \mathbf{a}_{-i}\right)\right) \propto q_{i, b_{i}} \exp \left(\beta \phi_{i}\left(b_{i}, \mathbf{a}_{-i}\right)\right)$. Similarly, $P\left(\left(b_{i}, \mathbf{a}_{-i}\right) \rightarrow\left(a_{i}, \mathbf{a}_{-i}\right)\right) \propto q_{i, a_{i}} \exp \left(\beta \phi_{i}\left(a_{i}, \mathbf{a}_{-i}\right)\right)$. The proportionality constants can be verified to be same. We also have, $\pi^{\prime}\left(a_{i}, \mathbf{a}_{-i}\right) \propto q_{i, a_{i}} \exp \left(\beta \phi_{i}\left(a_{i}, \mathbf{a}_{-i}\right)\right)$ and $\pi^{\prime}\left(b_{i}, \mathbf{a}_{-i}\right) \propto q_{i, b} \exp \left(\beta \phi_{i}\left(b_{i}, \mathbf{a}_{-i}\right)\right)$. This implies, $P\left(\left(a_{i}, \mathbf{a}_{-i}\right) \rightarrow\left(b_{i}, \mathbf{a}_{-i}\right)\right) \pi^{\prime}\left(\left(a_{i}, \mathbf{a}_{-i}\right)\right)=P\left(\left(b_{i}, \mathbf{a}_{-i}\right) \rightarrow\right.$ $\left.\left(a_{i}, \mathbf{a}_{-i}\right)\right) \pi^{\prime}\left(\left(b_{i}, \mathbf{a}_{-i}\right)\right)$, and therefore $\pi^{\prime}$ is the steady state distribution.
Thus, user associations with partial information gives rise to a modified Gibbs distribution, $\pi^{\prime}(\cdot)$. The next theorem shows that the optimality gap can be made arbitrarily small.

Theorem 2. Let $\bar{U}^{\prime}$ be the expected value of the utility in steady state, then

$$
\bar{U}^{\prime} \geq \mathbf{O P T}-\frac{1}{\beta} \log \frac{\eta}{\eta^{\star}}
$$

where $\eta=\sum_{\mathbf{a} \in \mathcal{F}} \eta_{\mathbf{a}}$ and $\eta^{\star}=\sum_{\mathbf{a} \in \mathcal{F} \star} \eta_{\mathbf{a}}$.

Proof: For a fixed optimal solution, $\mathbf{a}^{\star} \in \mathcal{F}^{\star}$, consider $\mathbb{E}\left[e^{\beta\left(U\left(\mathbf{a}^{\star}\right)-U(\mathbf{a})\right)}\right]=\sum_{\mathbf{a} \in \mathcal{F}} \pi^{\prime}(\mathbf{a}) e^{\beta\left(U\left(\mathbf{a}^{\star}\right)-U(\mathbf{a})\right)}=$ $\frac{\eta}{\eta_{\mathbf{a}^{\star}}} \pi^{\prime}\left(\mathbf{a}^{\star}\right) \leq \frac{\eta}{\eta^{\star}}$. The inequality is shown by using a result similar to lemma (1) which shows, $\pi^{\prime}\left(\mathbf{a}^{\star}\right) \leq \lim _{\beta \rightarrow \infty} \pi^{\prime}\left(\mathbf{a}^{\star}\right)=$ $\frac{\eta_{a^{\star}}}{\eta^{\star}}$. Now, applying Jensen's inequality to the exponential function, we have, $e^{\beta\left(U\left(\mathbf{a}^{\star}\right)-\bar{U}^{\prime}\right)} \leq \mathbb{E}\left[e^{\beta\left(U\left(\mathbf{a}^{\star}\right)-U(\mathbf{a})\right)}\right] \leq \frac{\eta}{\eta^{\star}}$. Taking $\log$ on both sides gives the result in the theorem.

The value of $\beta$ can be set to upperbound $\mathbf{O P T}-\bar{U}^{\prime}$. Choosing a large $\beta$ will, however, increase the time required for the Markov chain to reach steady state. Hence, $\beta$ represents a tradeoff between convergence and optimality.

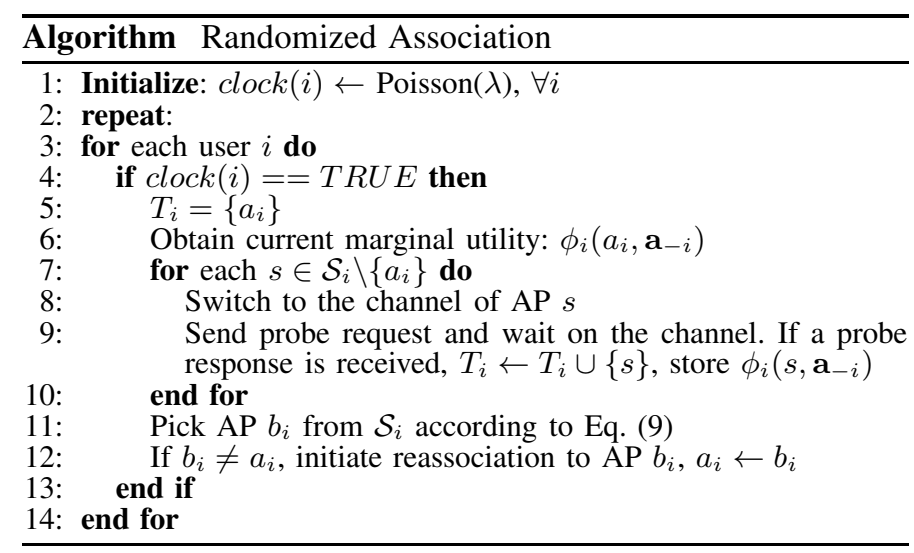

As summarized in the algorithm above, we have shown that the randomized solution to the user association problem is fully distributed. It is also robust to the scenarios of partial scanning and probe frame losses. Moreover, we get nearly optimal user associations, though, at the cost of slow convergence.

\section{Best Association}

While the randomized approach ensures that all states in the feasible set $\mathcal{F}$ are visited and selected probabilistically, there is no upper bound on the number of reassociations or the time it takes for users to reach steady state. This could be undesirable due to the frequent disruptions in the network caused by user reassociations. Also, user arrivals and departures need to be considered at this time scale. Next, we describe a greedy approach to randomization, a Best Association rule, that is suboptimal but converges quickly.

Assume that when a clock of user $i$ ticks, instead of picking an AP at random according to Eq. (8), it chooses the next AP $b_{i} \in \mathcal{S}_{i}$ that maximizes the marginal utility function, i.e.,

$$
b_{i}=\operatorname{argmax}_{s \in \mathcal{S}_{i}} \phi_{i}\left(s, \mathbf{a}_{-i}\right)
$$


A switch from AP $a_{i}$ to AP $b_{i}$ occurs if and only if $\phi_{i}\left(b_{i}, \mathbf{a}_{-i}\right)>\phi_{i}\left(a_{i}, \mathbf{a}_{-i}\right)$. Ties are broken randomly when the current AP is not involved. In contrast to the previously discussed probabilistic model, the AP selection is simple and deterministic. Moreover, this heuristic is intuitive for users and is easily realized in practice. In place of the default received signal strength index (RSSI) of an AP, we consider a similar metric based on the marginal utility of the user w.r.t. that AP. A user simply picks an AP iteratively that maximizes this index until no such AP exists.

From a game-theoretic viewpoint, the system can be analyzed as a non-cooperative game [20]. The strategy space of user $i \in \mathcal{N}$ is the set of APs $\mathcal{S}_{i}$ it can choose to associate with, and the reward for choosing AP $b_{i}$, for a given association choices of other users, $\mathbf{a}_{-i}$, is its marginal utility $\phi_{i}\left(b_{i}, \mathbf{a}_{-i}\right)$. The next result shows that the Best Association approach will lead to a Nash equilibrium, denoted by $\overline{\mathbf{a}} \in \mathcal{F}$, which satisfies the property that no user switches unilaterally to increase its marginal utility i.e., $\phi_{i}\left(\bar{a}_{i}, \overline{\mathbf{a}}_{-i}\right) \geq \phi_{i}\left(s, \overline{\mathbf{a}}_{-i}\right)$ for all $s \in \mathcal{S}_{i}$ and all $i \in \mathcal{N}$.

\section{Theorem 3. The Best Association converges almost surely.}

Proof: No two users switch at the same time. Let a single user $i$ selected switch its association from $\mathrm{AP} a_{i}$ to $\mathrm{AP} b_{i}$. It follows that $\phi_{i}\left(b_{i}, \mathbf{a}_{-i}\right)>\phi_{i}\left(a_{i}, \mathbf{a}_{-i}\right)$, which implies using Eq. (7), $U_{b_{i}}\left(n_{b_{i}}\left(b_{i}, \mathbf{a}_{-i}\right)\right)-U_{b_{i}}\left(n_{b_{i}}\left(\mathbf{a}_{-i}\right)\right)>U_{a_{i}}\left(n_{a_{i}}\left(a_{i}, \mathbf{a}_{-i}\right)\right)-$ $U_{a_{i}}\left(n_{a_{i}}\left(\mathbf{a}_{-i}\right)\right)$ and after rearranging, $U\left(b_{i}, \mathbf{a}_{-i}\right)>U\left(a_{i}, \mathbf{a}_{-i}\right)$. Therefore, the objective function improves strictly at every reassociation. Since the objective function is bounded above, there are only a finite number of increments. Thus, the system should converge in finite time almost surely.

Corollary 3.1. Every optimal association $\mathbf{a} \in \mathcal{F}^{\star}$ is an equilibrium association.

Corollary 3.2. The Best Association with partial scanning also converges almost surely.

We conclude that it takes at most $|\mathcal{F}|$ steps for the system to converge. Also, the equilibrium association $\overline{\mathbf{a}}$ corresponds to the local maximum in $\mathcal{F}$ of the global utility $U(\mathbf{a})$. Since there are many such equilibrium states depending on the update sequence of the users, it is highly likely the system will converge to a state that is far from optimal. Providing bounds on the resulting utility $U(\overline{\mathbf{a}})$ is difficult and next, we analyze two tractable cases of interest.

\section{A. Dense networks}

We consider a dense network deployment where users are located nearby and can connect to any AP, i.e., $\mathcal{S}_{i}=\mathcal{S}$ and $B_{i, s}=B_{s}$ for all users $i \in \mathcal{N}$ and APs $s \in \mathcal{S}$. This can happen, for instance, in a deployment of WiFi network within an apartment complex. Let $n:=\left(n_{1}, \ldots, n_{S}\right)$ represent the number of users associated with APs, $s=1,2 \ldots, S$, respectively so that $\sum_{s=1}^{S} n_{s}=N$. To facilitate the analysis, assume the overhead term is a linear function of the form, $O\left(n_{s}\right)=k_{1} n_{s}+k_{2}$, for some constants $k_{1}, k_{2} \geq 0$. Since the exact identity of the users is irrelevant here, with slight abuse of notation, the global utility is expressed as $U(n)=$ $\sum_{s \in \mathcal{S}} U_{s}\left(n_{s}\right)$. Obviously, $U_{s}(0)=0$ for AP $s$ that has no user. The marginal utility w.r.t. a AP $s$ for a user not associated with that $\mathrm{AP}$ is $\phi_{s}\left(n_{s}\right)=U_{s}\left(n_{s}+1\right)-U_{s}\left(n_{s}\right)$, while the marginal utility of users associated with $\mathrm{AP} s$ is $\phi_{s}\left(n_{s}-1\right)$. An equilibrium state, $\bar{n}=\left(\bar{n}_{1}, \ldots, \bar{n}_{S}\right)$, then satisfies the condition: $\phi_{s}\left(\bar{n}_{s}-1\right) \geq \phi_{l}\left(\bar{n}_{l}\right)$ for all $s, l \in \mathcal{S}$. We show that in this simple case, the Best Association rule leads to the optimal association of users. First, we show a couple of simple results that will be required in our proof.

Lemma 2. For $a, W>0$ and $b \geq 0$, the function $f(x)=$ $x \log \frac{W}{a x+b}$ defined on $\mathbb{R}_{++}$is concave.

Proof: See Appendix.

Lemma 3. For two concave functions $f$ and $g$, their minimum, $h=\min \{f, g\}$ is also concave.

Proof: See Appendix.

Theorem 4. Every equilibrium association $\bar{n}$ is globally optimal, that is $U(\bar{n})=\mathbf{O P T}$

Proof: From the throughput expression in Eq. (2), the utility associated with an $\mathrm{AP} s \in \mathcal{S}$ takes the form $U_{s}\left(n_{s}\right)=n_{s} \log \left\{\min \left(\frac{f_{s}}{\frac{n_{s}}{B_{s}}+k_{1} n_{s}+k_{2}}, \frac{W_{s}}{n_{s}}\right)\right\}=$ $\min \left\{n_{s} \log \frac{f_{s}}{\frac{n_{s}}{B_{s}+k_{1} n_{s}+k_{2}}}, n_{s} \log \frac{W_{s}}{n_{s}}\right\}$, since $\log$ is monotonic increasing. From lemma (2), both functions in the expression are concave in $n_{s}$, and from lemma (3), $U_{s}\left(n_{s}\right)$ is also concave. Consequently, $\phi_{s}\left(n_{s}\right)$ is monotonic decreasing for all $n_{s} \geq 0$ and $s \in \mathcal{S}$. We show that all equilibrium states have the same value of the utility function. For any two distinct equilibrium states, $\bar{n}=\left(\bar{n}_{1}, \ldots, \bar{n}_{S}\right)$ and $\bar{m}=\left(\bar{m}_{1}, \ldots, \bar{m}_{S}\right)$, take any indices $s, l \in\{1,2 \ldots S\}$ such that $\bar{n}_{s}>\bar{m}_{s}$ and $\bar{n}_{l}<\bar{m}_{l}$. From the equilibrium condition, $\phi_{s}\left(\bar{n}_{s}-1\right) \geq \phi_{l}\left(\bar{n}_{l}\right)$. Furthermore, the decreasing property of the marginal utility implies $\phi_{s}\left(\bar{m}_{s}\right) \geq \phi_{s}\left(\bar{n}_{s}-1\right) \geq \phi_{l}\left(\bar{n}_{l}\right) \geq \phi_{l}\left(\bar{m}_{l}-1\right)$. But for the equilibrium state $\bar{m}$, we have $\phi_{l}\left(\bar{m}_{l}-1\right) \geq \phi_{s}\left(\bar{m}_{s}\right)$. Therefore, $\bar{n}_{s}=\bar{m}_{s}+1, \bar{n}_{l}=\bar{m}_{l}-1$ and $\phi_{s}\left(\bar{n}_{s}-1\right)=\phi_{l}\left(\bar{n}_{l}\right)$ for all such $s$ and $l$. In addition, the number of APs $s$ that satisfy $\bar{n}_{s}=\bar{m}_{s}+1$ equals the number of APs $l$ with $\bar{n}_{l}=\bar{m}_{l}-1$. Now, consider $\Delta U=U(\bar{n})-U(\bar{m})=$ $\sum_{s: \bar{n}_{s}>\bar{m}_{s}}\left\{U_{s}\left(\bar{n}_{s}\right)-U_{s}\left(\bar{m}_{s}\right)\right\}+\sum_{l: \bar{n}_{l}<\bar{m}_{l}}\left\{U_{l}\left(\bar{n}_{l}\right)-U_{l}\left(\bar{m}_{l}\right)\right\}$. This further simplifies to $\Delta U=\sum_{s, l}\left(\phi_{s}\left(\bar{n}_{s}-1\right)-\phi_{l}\left(\bar{n}_{l}\right)\right)=0$ for all equilibrium states $\bar{n}$ and $\bar{m}$. Together with the corollary 3.1 , this proves the claim.

\section{B. Backhaul-limited}

We analyze scenarios when the backhaul capacity of APs becomes the bottleneck in the network. This may occur when there are a few users in network, all of whom are connected to APs at high link rates or when the backhaul bandwidth is just too low. From Eq. (2), the throughput of user $i \in \mathcal{N}$ in this case is only a function of number of users $\left|n_{a_{i}}(\mathbf{a})\right|$ sharing its AP. Again, let the state of the system be represented by $n=\left(n_{1}, \ldots, n_{S}\right)$. The equilibrium state, $\bar{n}=\left(\bar{n}_{1}, \ldots, \bar{n}_{S}\right)$, 
for any two APs $s, l \in \mathcal{S}$ satisfies $\phi_{s}\left(\bar{n}_{s}-1\right) \geq \phi_{l}\left(\bar{n}_{l}\right)$ if a user connected to AP $s$ can also associate with AP $l$. In contrast to the previous case, the users can be spread across the network and connect to only a subset of all APs. However, we can precisely bound the worst-case value of an equilibrium state.

Theorem 5. If all APs are backhaul-limited and the units of the backhaul bandwidth are taken so that $W_{s} \geq N e$ for all $s \in \mathcal{S}$, then $U(\bar{n}) \geq \frac{1}{2} \mathbf{O P T}$.

Proof: The function $U_{s}\left(n_{s}\right)=n_{s} \log \frac{W_{s}}{n_{s}}$ is strictly increasing and concave (lemma (2)) in $\left(0, W_{s} / e\right]$. Given the condition, $W_{s} \geq N e$, it implies that the utility of an AP, $U_{s}\left(n_{s}\right)$, is an increasing function while the marginal utility, $\phi_{s}\left(n_{s}\right)$, is monotonic decreasing and satisfies $\phi_{s}\left(n_{s}\right)>0$ for all $n_{s} \leq N$ and $s \in \mathcal{S}$. Let $n^{\star}=\left(n_{1}^{\star}, \ldots, n_{S}^{\star}\right)$ be the optimal configuration of users and let $\mathcal{S}^{\prime}=\left\{s \mid n_{s}^{\star}>\bar{n}_{s}\right\}$. Then, for any $s \in \mathcal{S}^{\prime}$, we can write $U_{s}\left(n_{s}^{\star}\right)-U_{s}\left(\bar{n}_{s}\right)=n_{s}^{\star} \log \frac{W_{s}}{n_{s}^{\star}}-\bar{n}_{s} \log \frac{W_{s}}{\bar{n}_{s}}=$ $\sum_{m=\bar{n}_{s}^{\star}-1}^{m} \phi_{s}(m) \leq\left(n_{s}^{\star}-\bar{n}_{s}\right) \phi_{s}\left(\bar{n}_{s}\right)$. Therefore, $U\left(n^{\star}\right)-$ $U(\bar{n}) \leq \sum_{s \in \mathcal{S}^{\prime}}\left(U_{s}\left(n_{s}^{\star}\right)-U_{s}\left(\bar{n}_{s}\right)\right) \leq \sum_{s \in \mathcal{S}^{\prime}}\left(n_{s}^{\star}-\bar{n}_{s}\right) \phi_{s}\left(\bar{n}_{s}\right)$. Now, since $\bar{n}$ is an equilibrium state, for each AP $s \in \mathcal{S}^{\prime}$, $n_{s}^{\star}-\bar{n}_{s}$ users did not switch to that AP $s$. It means $\phi_{s}\left(\bar{n}_{s}\right) \leq$ $\phi_{l}\left(\bar{n}_{l}-1\right)$ for some AP $l \neq s$ for each of those users. Therefore, we can write $\sum_{s \in \mathcal{S}^{\prime}}\left(n_{s}^{\star}-\bar{n}_{s}\right) \phi_{s}\left(\bar{n}_{s}\right) \leq \sum_{s \in \mathcal{S}} \bar{n}_{s} \phi_{s}\left(\bar{n}_{s}-1\right)$. Now, use the inequality $\phi_{s}\left(n_{s}-1\right)=n_{s} \log \frac{W_{s}}{n_{s}}-\left(n_{s}-\right.$ 1) $\log \frac{W_{s}}{n_{s}-1} \leq n_{s} \log \frac{W_{s}}{n_{s}}-\left(n_{s}-1\right) \log \frac{W_{s}}{n_{s}}=\log \frac{W_{s}}{n_{s}}$ for all $n_{s} \geq 1$. As a result, $U\left(n^{\star}\right)-U(\bar{n}) \leq \sum_{s \in \mathcal{S}} \bar{n}_{s} \log \frac{W_{s}}{\bar{n}_{s}}=U(\bar{n})$ or $U(\bar{n}) \geq \frac{1}{2} U\left(n^{\star}\right)$.

\section{User dynamics}

As stated earlier, the upper bound on the number of reassociations is $|\mathcal{F}|$. This is also true when the number of users, $N$ in the network changes due to arrivals and departures. Here, we consider the previous scenario of a dense network deployment in section IV.A and show that the equilibrium state is reached much more easily. The system state is given by $n=\left(n_{1}, \ldots, n_{S}\right)$ and $\phi_{s}\left(n_{s}\right)$ is a decreasing function for $n_{s} \geq 0$. A user arriving in the system follows the Best Association rule while users leaving the system can depart from any AP.

Theorem 6. Arrival of a new user maintains the equilibrium.

Proof: Let $\bar{n}$ be the Nash equilibrium state and assume that a new user chooses AP $s$ such that $\phi_{s}\left(\bar{n}_{s}\right) \geq \phi_{l}\left(\bar{n}_{l}\right), \forall l \neq s$. For users associated with AP $s$, the new marginal utility satisfies $\phi_{s}\left(\left(\bar{n}_{s}+1\right)-1\right)=\phi_{s}\left(\bar{n}_{s}\right) \geq \phi_{l}\left(\bar{n}_{l}\right)$ for all $l \neq s$. For users associated with other APs, $\phi_{l}\left(\bar{n}_{l}-1\right) \geq \phi_{s}\left(\bar{n}_{s}\right) \geq \phi_{s}\left(\bar{n}_{s}+1\right)$ for all $l \neq s$. Collectively, this shows that no user can further improve its marginal utility.

Also, we show that the maximum number of reassociations needed from any starting state is $N$, and therefore gives us the next theorem.

Theorem 7. A user departing will lead to at most $N-1$ reassociations.
Proof: We provide a sketch of the proof. We claim that once a user switches to an AP, users from that AP will not switch later. Let the current state of the system be $n=\left(n_{1}, \ldots, n_{S}\right)$ and consider the next switch of a user between APs $r, s \in \mathcal{S}$ as $r \rightarrow s$. We show by induction that $s \not \rightarrow t$ for any later time instant for any AP $t \neq s$. Consider the base case and assume next switch is given by, $k \rightarrow l$, and $s \rightarrow t$ occurs after this. Clearly, $k \neq s$ and we only worry when $t$ is either $l$ or $k$. Consider three cases: (i) $l \neq s, k \neq r$, the users with AP $s$ after the second switch will not switch to $k$ because $\phi_{s}\left(n_{s}\right) \geq \phi_{l}\left(n_{l}\right)$ and $\phi_{l}\left(n_{l}\right) \geq \phi_{k}\left(n_{k}-1\right)$ from two switch conditions and therefore, $\phi_{s}\left(n_{s}\right) \geq \phi_{k}\left(n_{k}-1\right)$. (ii) $l=s$, users from $s$ wont switch to $t$, because user from $k$ did not switch to $t$ (iii) $k=r$, users from AP $s$ will not switch to AP $r$ because $\phi_{s}\left(n_{s}\right) \geq \phi_{l}\left(n_{l}\right)>\phi_{r}\left(n_{r}-2\right)$. Now, from the induction step, assume $s \rightarrow t$ is not possible at time $T$, and instead switch occurs as $k \rightarrow l$. It is then easy to show that $s \rightarrow t$ is not feasible for time $T+1$. Therefore, the claim is true for all $T$. This implies that each user will switch at most once to reach equilibrium. Hence there at most $N-1$ reassociations when a user departs.

In summary, the Best Association policy is based on the greedy version of the randomized solution. It is suboptimal, but simple and fast converging; we showed its performance in various special cases.

\section{Evaluation}

In this section, we present the evaluation (via simulation) results of the proposed association control mechanisms. We restrict the evaluation to the MAC layer and do not study the association overhead at higher layers. For the purpose of comparison, we take the two commonly used performance measures: (i) average user throughput $\bar{r}=\left(\sum_{i} r_{i}\right) / N$ and (ii) Jain's fairness index $(\mathrm{JFI}) \triangleq\left(\sum_{i} r_{i}\right)^{2} /\left(N \sum_{i} r_{i}^{2}\right)$, that varies from $1 / N$ (least fair) to 1 (equal throughputs).

\section{A. Numerical Evaluation}

We consider a deployment of overlapping APs placed randomly in each of the 9 grids in a $300 m \times 300 m$ square area. The backhaul capacity of each is set to $10 \mathrm{Mbps}$. There are 50 users randomly deployed in this area following a uniform distribution. The range of AP is taken to be $200 \mathrm{~m}$ and users' physical rates are selected based on distance between APs and the users following the $802.11 \mathrm{~g}$ specification in [21]. Each AP is assigned one of the three orthogonal channels in the 2.4 $\mathrm{GHz}$ band, and assuming fair access to channel, the fraction $f_{s}$ is set to $1 / 3$ for all $s$. We assume a linear overhead term $O\left(n_{s}\right)=0.0171 n_{s}$ so that the throughput at 54Mbps link rate is equal to the observed $28 \mathrm{Mbps}$ for a single user. The mean scanning period of users is set to $1 / \lambda=120$ seconds.

Fig. 3 shows the simulation results of a single run for the three proposed solutions with a run duration of $10^{4}$ seconds. Two values of $\beta$ are chosen to show its effect on convergence. The initial association of each user is set to its closest AP following the best RSSI strategy. In the partial randomized 


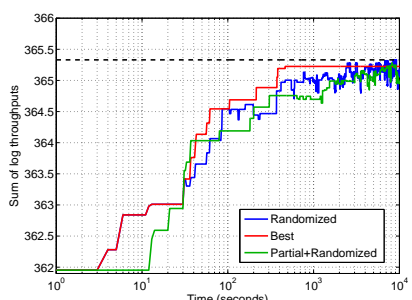

(a) $\beta=20$

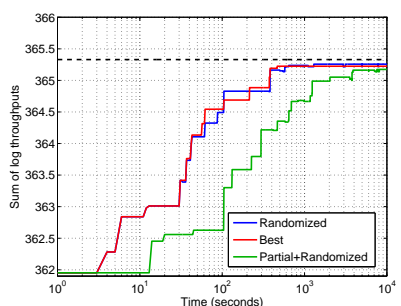

(b) $\beta=80$
Fig. 3. Simulation results shows the effect of $\beta$ on proposed solutions for a random deployment of 9 APs and 50 users

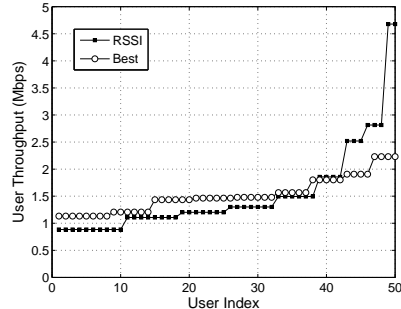

(a)

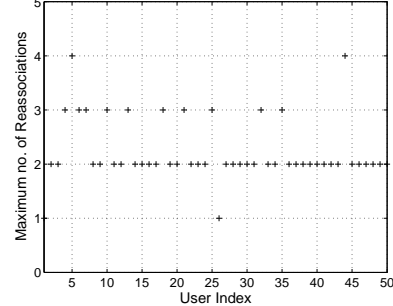

(b)
Fig. 4. (a) Throughput performance before and after association control (b) Maximum number of reassociations needed for users to reach steady state

method, user $i$ only probes two randomly chosen APs in $\mathcal{S}_{i}$. The user associations were changed following Eqs. (8), (9) and (12) for the three cases, respectively. The changes in trajectory of the objective function in Fig. 4a correspond to change in association of a user. The Best Association strictly increases the objective function and thus converges. The randomized solutions take longer to reach steady state, with partial randomized being the slowest. We observe that close to optimal states are reached around the $10^{3}$ seconds mark. Also, the Best Association gives nearly optimal solution: $U(\overline{\mathbf{a}})=365.22$ and OPT $=365.33$. We see with $\beta=80$, there are fewer changes in user associations, the expected utility is more close to optimal and it takes longer to reach steady state. The partial update mechanism takes the longest time to converge in both cases. Intuitively, this presents a tradeoff between optimality, speed of convergence and reassociation overhead.

The plot in Fig. 4a shows sorted user throughputs before and after the Best Association rule. One can observe that initial associations based on RSSI led to skewed distribution of user throughputs. The initial values of average throughput $\bar{r}=1.53 \mathrm{Mbps}$ and JFI $=0.7727$ changed to $\bar{r}=1.51 \mathrm{Mbps}$ and JFI $=0.9509$ after association control. This improvement in performance is from the proportional fair (PF) utility used, which improves fairness when the sum throughput is roughly kept constant. Fig. $4 \mathrm{~b}$ shows the maximum reassociation overhead in the Best Association over a number of runs. At most, 4 reassociations were needed by users and on an average most users had to switch only twice.

In Fig. 5a, we plot the utility function for a dense network deployment where all users can connect to any AP and do so at the same PHY rates. As shown previously, the greedy association rule gives the optimal user associations. One can see

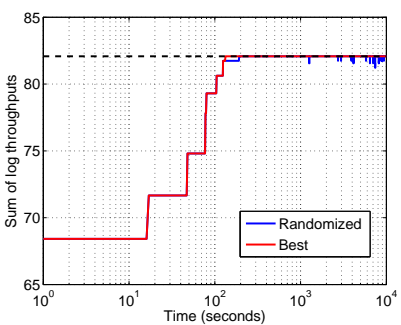

(a)

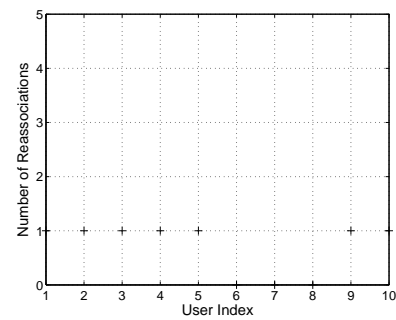

(b)
Fig. 5. Evaluation of Best Association in Dense network deployment with 10 users and 4 APs. Plots showing that equilibrium association is optimal (Theorem (4)) and the number of reassociations per user to reach equilibrium (Theorem (7))

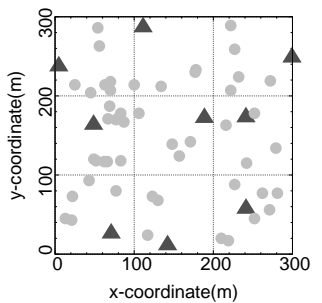

(a) Unfair topology

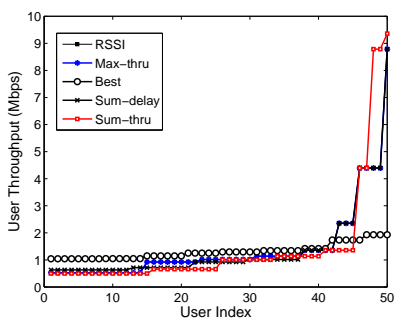

(b) User throughputs
Fig. 6. Comparison of our metric with other distributed solutions with an unfair network topology

that there are perturbations in associations in the randomized solution even after steady state is reached. Also, each user needs to reassociate at most once to reach equilibrium.

\section{B. Comparison}

We implement and compare our Best Association solution with other distributed AP association rules. Specifically, we consider: Sum-delay [13], that tries to minimize the sum of user packet delays, Max-thru [8], where each user greedily maximizes its own throughput, and Sum-thru [22], where users choose APs so as to maximize the overall throughput in the system. We modified their expressions to include the additional overhead term and backhual constraint.

We run these association policies with an unfair topology example shown in Fig. 6a. Clearly, some of the APs (triangles) are overloaded from users (circles) by following the default RSSI rule. Fig. 6b shows the averaged throughput distrbution across the users for the different metrics used. As seen, the default RSSI led to associations where an AP served a single user at $9 \mathrm{Mbps}$ while more than 30 users received $0.4 \mathrm{Mbps}$.

Table 1 lists the average performance metrics over 10 runs. Maximizing individual throughput achieves reasonable fairness while being the least efficient. This is attributed to the loadbalancing of APs [8]. Sum-delay only minimizes the packet transmission times and therefore has no notion of fairness. Sum-thru gives the highest throughput in the system while being the most inefficient in distribution of throughput. In comparison, Best Association, based on proportional fairness, outperformed on the fairness measure with a slight reduction in 
TABLE I

COMPARISON OF DIFFERENT DISTRIBUTED AP-ASSOCIATION POLICIES AVERAGED OVER SIMULATION RUNS

\begin{tabular}{|c|c|c|c|}
\hline Utility & Fairness Index & Mean thru. (Mbps) & Total switches \\
\hline \hline RSSI & 0.48 & 1.42 & 0 \\
\hline Best-Assoc & 0.96 & 1.32 & 28 \\
\hline Sum-delay [13] & 0.47 & 1.39 & 3 \\
\hline Max-thru [8] & 0.77 & 0.68 & 34 \\
\hline Sum-thru [22] & 0.33 & 1.45 & 381 \\
\hline
\end{tabular}

total throughput (1.32 Mbps vs $1.42 \mathrm{Mbps}$ with RSSI). Also, the reassociation overhead is reasonable in this case.

Based on these observations, we conclude that the Best Association performs close to optimal, providing both fairness and throughput-efficiency .

\section{CONCLUding REMARKS}

We have presented and solved the problem of distributed association control that arises in many modern WLAN deployments. We derived a provably optimal solution, which is completely distributed and can be practically implemented by the mobile users. We have also proposed a greedy heuristic, Best Association, based on the concept of marginal utility. In our simulation results, the Best Association rule gives almost optimal solutions and has a low reassociation overhead.

Some of the assumptions made in our work can be relaxed in practice. In particular, the update process need not follow a Poisson clock. The convergence of the greedy heuristic will still hold. However, the strict assumption of cooperative users is required to solve for the optimal user associations.

\section{APPENDIX}

Proof of Lemma 1: For $\mathbf{a} \in \mathcal{F}^{\star}$, taking the derivative of $\pi(\mathbf{a})$ in Eq. (5) w.r.t $\beta$,

$$
\begin{aligned}
\frac{\partial \pi(\mathbf{a})}{\partial \beta} & =\frac{U(\mathbf{a}) e^{\beta U(\mathbf{a})}}{\sum_{\mathbf{a}^{\prime} \in \mathcal{F}} e^{\beta U\left(\mathbf{a}^{\prime}\right)}-\frac{\sum_{\mathbf{a}^{\prime} \in \mathcal{F}} U\left(\mathbf{a}^{\prime}\right) e^{\beta U\left(\mathbf{a}^{\prime}\right)} e^{\beta U(\mathbf{a})}}{\left[\sum_{\mathbf{a}^{\prime} \in \mathcal{F}} e^{\beta U\left(\mathbf{a}^{\prime}\right)}\right]^{2}}} \\
& =\frac{\sum_{\mathbf{a}^{\prime} \in \mathcal{F}}\left[U(\mathbf{a})-U\left(\mathbf{a}^{\prime}\right)\right] e^{\beta U\left(\mathbf{a}^{\prime}\right)} e^{\beta U(\mathbf{a})}}{\left[\sum_{\mathbf{a}^{\prime} \in \mathcal{F}} e^{\beta U\left(\mathbf{a}^{\prime}\right)}\right]^{2}} \\
& \geq 0
\end{aligned}
$$

since $U(\mathbf{a}) \geq U\left(\mathbf{a}^{\prime}\right)$ for any $\mathbf{a} \in \mathcal{F}^{\star}$ and $\mathbf{a}^{\prime} \in \mathcal{F}$. Note that Eq. (5) can also be expressed as:

$$
\pi(\mathbf{a})=\frac{1}{\left|\mathcal{F}^{\star}\right|+\sum_{\mathbf{a}^{\prime} \in \mathcal{F} \backslash \mathcal{F} \star} e^{\beta\left(U\left(\mathbf{a}^{\prime}\right)-U(\mathbf{a})\right)}}
$$

which gives the result $\lim _{\beta \rightarrow \infty} \pi(\mathbf{a})=\frac{1}{\mathcal{F}^{\star} \mid}$ for $\mathbf{a} \in \mathcal{F}^{\star}$.

Proof of Lemma 2: The first and the second derivate of the function are given by:

$$
\begin{aligned}
f^{\prime}(x) & =\log \frac{W}{a x+b}-\frac{a x}{a x+b} \\
f^{\prime \prime}(x) & =\frac{-a^{2} x-2 a b}{(a x+b)^{2}}
\end{aligned}
$$

Clearly, $f^{\prime \prime}(x)<0$ for all $x>0$ and therefore, $f(x)$ is concave in $\mathbb{R}_{++}$.

Proof of Lemma 3: A function is concave if and only if the epigraph of the function is a concave region. The epigraph of the function $h=\min \{f, g\}$ is a union of concave regions above $f$ and $g$, and is thus concave. Hence $h$ is a concave function.

\section{ACKNOWLEDGEMENTS}

The work reported in this paper was supported in part by the National Science Foundation under Grant CNS-1160775.

\section{REFERENCES}

[1] "FON," http://www.fon.com.

[2] "Freifunk," http://freifunk.net.

[3] P. Frangoudis, G. Polyzos, and V. Kemerlis, "Wireless community networks: an alternative approach for nomadic broadband network access," In IEEE Communications Magazine, vol. 49, no. 5, pp. 206 -213, may 2011.

[4] E. C. Efstathiou, P. A. Frangoudis, and G. C. Polyzos, "Stimulating Participation in Wireless Community Networks," in Proceedings of INFOCOM, 2006.

[5] G. Athanasiou, T. Korakis, O. Ercetin, and L. Tassiulas, "A Cross-Layer Framework for Association Control in Wireless Mesh Networks," In IEEE Transactions on Mobile Computing, vol. 8, no. 1, pp. 65 -80, Jan. 2009.

[6] Y. Bejerano, S.-J. Han, and L. E. Li, "Fairness and load balancing in wireless LANs using association control," in Proceedings of MOBICOM, 2004.

[7] D. Giustiniano, E. Goma, A. Lopez, I. Dangereld, J. Morillo, and P. Rodriguez, "Fair WLAN Backhaul Aggregation," in Proceedings of MOBICOM, 2010.

[8] H. Gong, K. Nahm, and J. W. Kim, "Distributed Fair Access Point Selection for Multi-Rate IEEE 802.11 WLANs," in IEEE Consumer Communications and Networking Conference, 2008.

[9] J.-C. Chen, T.-C. Chen, T. Zhang, and E. van den Berg, "Effective AP Selection and Load Balancing in IEEE 802.11 Wireless LANs," in Proceedings of GLOBECOM, 2006.

[10] A. Kumar and V. Kumar, "Optimal association of stations and APs in an IEEE 802.11 Wlan.” in Proceedings of NCC, 2005.

[11] E. L. Li, M. Pal, and Y. R. Yang, "Proportional Fairness in Multi-Rate Wireless LANs," in Proceedings of INFOCOM, 2008.

[12] L. L. Tian Bu and R. Ramjee, "Generalized Proportional Fair Scheduling in Third Generation Wireless Data Networks," in Proceedings of INFOCOM, 2006

[13] B. Kauffmann, F. Baccelli, A. Chaintreau, V. Mhatre, K. Papagiannaki, and C. Diot, "Measurement-Based Self Organization of Interfering 802.11 Wireless Access Networks," in Proceedings of INFOCOM, 2007.

[14] I. Broustis, K. Papagiannaki, S. Krishnamurthy, M. Faloutsos, and V. Mhatre, "Measurement-Driven Guidelines for 802.11 WLAN Design," In IEEE/ACM Transactions on Networking, vol. 18, no. 3, pp. $722-735$, june 2010.

[15] P. Bremaud, Markov chains : Gibbs fields, Monte Carlo simulation and queues. New York, USA: Springer, 1999, ISBN: 0-387-98509-3.

[16] X. Xing, J. Dang, S. Mishra, and X. Liu, "A highly scalable bandwidth estimation of commercial hotspot access points," in Proceedings of INFOCOM, 2011

[17] M. Heusse, F. Rousseau, G. Berger-Sabbatel, and A. Duda, "Performance Anomaly of 802.11b," in Proceedings of INFOCOM, vol. 2, 2003.

[18] "IEEE 802.11-2007, Wireless LAN Medium Access Control (MAC) and Physical Layer (PHY) Specifications ," 2007.

[19] "MadWifi Open Source Driver," http://madwifi-project.org/.

[20] J. Nash, "Non-cooperative games," in Annals of Mathematics, 1951.

[21] "Cisco Aironet 1200 Data sheet," http://tinyurl.com/5nd616.

[22] M. Y. Arslan, K. Pelechrinis, I. Broustis, S. V. Krishnamurthy, S. Addepalli, and K. Papagiannaki, "Auto-configuration of 802.11n WLANs," in Proceedings of Co-NEXT, 2010. 